\title{
A Retrospective Study of the Optimal Number of Blood Cultures at a Hospital in Japan
}

\author{
Takehiro Hashimoto, ${ }^{1}$ Daisuke Shiojiri ${ }^{1}$ and Norio Ohmagari ${ }^{1}$ \\ ${ }^{1}$ Disease Control and Prevention Center, National Center for Global Health and Medicine, Tokyo, Japan
}

\begin{abstract}
Blood culture tests have a major role in rapid and accurate diagnosis and selection of optimal antibiotic treatment for infectious diseases. The true-positive and contamination rates of blood cultures are performance indicators for the practice of blood culture. However, the optimal number of blood cultures has not been adequately elucidated. We aimed to assess the validity of the number of blood cultures obtained in the hospital. We conducted a retrospective study of blood cultures collected from hospitalized patients between 2010 and 2014 at a tertiary care hospital in Japan and assessed the validity of the number of blood cultures per 1,000 admissions as an indicator for the optimal practice of obtaining blood culture, considering the average length of stay. We also investigated the validity of true-positive and contamination rates of blood cultures in the hospital. The number of blood cultures in this study ranged from 28.6 to 56 per 1,000 patient-days and from 404.4 to 894.6 per 1,000 admissions. The median truepositive and contamination rates of blood cultures per year was $10.1 \%$ and $2.8 \%$, respectively. The median contamination rate of blood cultures in a community-acquired group was significantly higher than that in a hospital-acquired group $(4.1 \%$ vs. $1.9 \%, p=0.029)$. We conclude that there is a possibility that the number of blood cultures per 1,000 admissions is a more appropriate factor to evaluate than per patient-days. Moreover, the true-positive and contamination rates of blood cultures in our hospital are valid.
\end{abstract}

Keywords: 1,000 admissions; blood cultures; contamination rates; 1,000 patient-days; true-positive rate Tohoku J. Exp. Med., 2021 April, 253 (4), 233-239.

\section{Introduction}

Blood cultures are commonly ordered in clinical practice, typically when physicians suspect a bloodstream infection. Guidelines for optimal taking of blood cultures, including timing and volume of blood collection and number of cultures obtained for each patient, have been proposed (Baron et al. 2005). Patient-days and positive rate of blood cultures are assessed as performance indicators for the practice of blood culture, making international comparisons possible (Ohmagari et al. 2012). However, the optimal number of blood cultures per 1,000 patient-days has not been adequately elucidated (Ohmagari et al. 2012). In Cumitech 1C, the number of blood cultures per 1,000 patient-days was recommended to be 103-188 in the USA (Baron et al. 2005). By contrast, in a previous study of six hospitals in Japan, including our hospital, this number was as low as 10.4-64.2 per 1,000 patient-days; thus it is thought that the number of blood cultures might be insufficient in hospitals in Japan (Ohmagari et al. 2012). However, this result should be evaluated with caution. There are important differences in patient background, average length of stay, and the medical insurance system between the USA and Japan (Kim et al. 2012). The average length of stay in our hospital in 2010 was 15.3 days compared with 4.5 days in the USA (Weiss et al. 2014). It is possible that this difference affects the number of blood cultures per 1,000 patient-days. Filius et al. (2005) reported that evaluation using patient-days is affected by hospital resource indicators, including length of stay. On the other hand, evaluation using the number of admissions is not so easily affected, and thus the number of blood cultures per 1,000 admissions may not be affected by the average length of stay.

Moreover, it is possible that there are difficulties in assessing the optimal number of blood cultures because of a low threshold for physicians to collect them. It is reported that blood cultures are overutilized and a significant number of patients at little to no risk of bloodstream infections have

Received January 6, 2021; revised and accepted February 22, 2021. Published online April 2, 2021; doi: 10.1620/tjem.253.233.

Correspondence: Takehiro Hashimoto, Disease Control and Prevention Center, National Center for Global Health and Medicine, 1-21-1

Toyama, Shinjuku-ku, Tokyo 162-8655, Japan.

e-mail: hashimo2013@oita-u.ac.jp

(C)2021 Tohoku University Medical Press. This is an open-access article distributed under the terms of the Creative Commons Attribution-NonCommercial-NoDerivatives 4.0 International License (CC-BY-NC-ND 4.0). Anyone may download, reuse, copy, reprint, or distribute the article without modifications or adaptations for non-profit purposes if they cite the original authors and source properly.

https://creativecommons.org/licenses/by-nc-nd/4.0/ 
blood cultures taken (Bate et al. 1990). Therefore, the truepositive and contamination rates of blood cultures are assessed as part of performance indicators for the practice of blood culture (Baron et al. 2005; Nannan Panday et al. 2019; Halstead et al. 2020). Although several studies reported the true-positive and contamination rates of blood cultures in hospital settings, these rates vary (Baron et al. 2005; Chen et al. 2018; Nannan Panday et al. 2019). There are few studies comparing collecting blood cultures during hospitalization (Nannan Panday et al. 2019), and the change of true-positive and contamination rates of blood cultures during hospitalization has not been adequately elucidated.

Therefore, the objective of this study was to obtain a better performance indicator for the practice of blood culture, and to assess the validity of the true-positive and contamination rates of blood cultures obtained in the hospital.

\section{Materials and Methods}

We conducted a retrospective study of blood cultures collected from hospitalized patients between January 2010 and December 2014 at the National Centre for Global Health and Medicine (NCGM), Tokyo, Japan. NCGM has more than 780 inpatient beds and serves as a tertiary referral hospital for metropolitan Tokyo. Blood culture data were extracted from hospital electronic databases and case records, including date of admission and date of collecting blood cultures. The basic hospital statistics, including total number of admissions, total number of discharges, total number of patient-days, and average length of stay were obtained from the hospital administration department.

One set of blood cultures was defined as the blood obtained by one vessel puncture, which was divided between one aerobic bottle and one anaerobic bottle. A single blood vessel puncture, with the blood divided among three or more bottles, was still regarded as one set of blood culture (Novis et al. 2001). A true-positive blood culture was defined as the isolation of an organism generally considered to be a pathogen. A contaminated blood culture was considered as contaminated if the following organisms were identified in only one of a series of blood culture specimens: Coagulase-negative Staphylococci (CNS), Corynebacterium spp., Propionibacterium acnes, Bacillus spp., viridans group Streptococci and Micrococcus spp. (Baron et al. 2005). A community-acquired group was defined as a true-positive blood culture or contaminated blood culture within 48 hours after admission. A hospitalacquired group was defined as a true-positive blood culture or contaminated blood culture more than 48 hours after admission. Included participants were those admitted to our hospital from January 2010 to December 2014. Participants whose blood was not collected during hospitalization were excluded. The number of blood cultures per 1,000 patient-days was defined as (total number of blood cultures/number of patient-days) $\times 1,000$; the number of patient-days as average length of stay $\times\{$ (admissions + discharges) $/ 2\}$; the number of blood cultures per 1,000 admis- sions as (total number of blood cultures/admissions) $\times$ 1,000 , as per the definition by the Ministry of Health, Labour and Welfare of the Japanese government. The truepositive rate of blood cultures was defined as the number of true-positive blood cultures/number of blood cultures. The contamination rate of blood cultures was defined as the number of contaminated blood cultures/number of blood cultures. The cumulative incidence of number of blood cultures was defined as the total number of blood cultures collected since admission. The cumulative incidence of truepositive blood cultures was defined as the total number of true-positive blood cultures obtained since admission. The cumulative incidence rate of blood cultures was defined as cumulative incidence of true-positive blood cultures/cumulative incidence of number of blood cultures.

To obtain a better performance indicator for the practice of blood culture, we assessed the validity of evaluation based on the number of blood cultures per 1,000 admissions, taking into consideration the average length of stay. Moreover, we analysed the validity and change of the truepositive and contamination rates of blood cultures during hospitalization.

Data were analysed using Microsoft Excel 2013 and EZR (Saitama Medical Centre, Jichi Medical University, Saitama, Japan), which is a graphical user interface for $\mathrm{R}$ (The R Foundation for Statistical Computing, Vienna, Austria, version 2.13.0) (Kanda 2013). Bivariate analyses were performed using Student's $t$ test for the comparison of mean values between two samples. $P$ values of $<0.05$ were considered to indicate statistical significance. This study was approved by the Human Research Ethics Committee of NCGM (NCGM-G-003106-00) prior to its initiation.

\section{Results}

The median number of blood cultures per year obtained at the NCGM from January 2010 to December 2014 was 10,252 sets (range 9,242-11,257). The median true-positive rate of blood cultures per year was $10.1 \%$ (range 8.4-11.9\%). The median contamination rate of blood cultures per year was $2.8 \%$ (range $2.2-2.9 \%$ ). The median number of admissions per year was 15,936 (range 15,08717,259 ), and the median number of discharges per year was 15,914 (range 15,161-17,184). The average length of stay was 15.3 days in 2010 . The number of blood cultures in this study ranged from 28.6 to 56 per 1,000 patient-days and from 404.4 to 894.6 per 1,000 admissions.

The total number of blood cultures for hospitalized patients in 2014 was 8,440 sets collected from 3,914 patients (Table 1). The number of true-positive, contaminated and negative blood cultures was 932 sets, 240 sets and 7,268 sets, respectively. The number of true-positive blood cultures obtained on the day of admission was 479 sets, which composed $51.4 \%$ of the total number of positive blood cultures (932 sets) obtained during hospitalization; the rate of blood culture collection decreased gradually after the day of admission. The number of blood cultures 
Table 1. Summaries of blood cultures of hospitalized patients at the National Centre for Global Health and Medicine, Japan in 2014.

\begin{tabular}{|c|c|c|c|c|}
\hline $\begin{array}{l}\text { Time from admission } \\
\text { (days) }\end{array}$ & $\begin{array}{l}\text { True-positive blood } \\
\text { cultures } \\
\text { (sets) }\end{array}$ & $\begin{array}{l}\text { Contaminated blood } \\
\text { cultures } \\
\text { (sets) }\end{array}$ & $\begin{array}{l}\text { Negative blood } \\
\text { cultures } \\
\text { (sets) }\end{array}$ & $\begin{array}{l}\text { Total blood } \\
\text { cultures } \\
\text { (sets) }\end{array}$ \\
\hline 1 & 479 & 139 & 1,803 & 2,421 \\
\hline 2 & 7 & 9 & 337 & 353 \\
\hline 3 & 33 & 5 & 199 & 237 \\
\hline 4 & 13 & 1 & 164 & 178 \\
\hline 5 & 17 & 5 & 172 & 194 \\
\hline 6 & 20 & 6 & 158 & 184 \\
\hline 7 & 11 & 4 & 128 & 143 \\
\hline 8 & 17 & 5 & 133 & 155 \\
\hline 9 & 21 & 0 & 120 & 141 \\
\hline 10 & 16 & 3 & 84 & 103 \\
\hline 11 & 4 & 3 & 90 & 97 \\
\hline 12 & 3 & 1 & 85 & 89 \\
\hline 13 & 4 & 2 & 85 & 91 \\
\hline 14 & 10 & 3 & 74 & 87 \\
\hline 15 & 20 & 3 & 75 & 98 \\
\hline 16 & 2 & 2 & 68 & 72 \\
\hline 17 & 13 & 2 & 73 & 88 \\
\hline 18 & 9 & 0 & 61 & 70 \\
\hline 19 & 6 & 1 & 62 & 69 \\
\hline 20 & 3 & 1 & 52 & 56 \\
\hline 21 & 10 & 1 & 39 & 50 \\
\hline 22 & 14 & 1 & 59 & 74 \\
\hline 23 & 11 & 2 & 47 & 60 \\
\hline 24 & 4 & 0 & 50 & 54 \\
\hline 25 & 3 & 2 & 48 & 53 \\
\hline 26 & 2 & 1 & 28 & 31 \\
\hline 27 & 3 & 0 & 37 & 40 \\
\hline 28 & 0 & 0 & 37 & 37 \\
\hline 29 & 0 & 0 & 41 & 41 \\
\hline$>30$ & 177 & 38 & 2,859 & 3,074 \\
\hline Total (sets) & 932 & 240 & 7,268 & 8,440 \\
\hline
\end{tabular}

collected on the day of admission and after 30 days of hospitalization accounted for $28.7 \%$ and $36.4 \%$ of the total number of blood cultures $(8,440$ sets $)$ during hospitalization, respectively (Fig. 1). The number of blood cultures obtained each day during hospitalization exhibited skewed distribution, with a peak on the day of admission, as well as a distribution with a long tail after 30 days of hospitalization (Fig. 1). The cumulative incidence rate of true-positive blood cultures was highest at day of admission (19.8\%), decreasing to $16.0 \%$ up to day 6 and to $14.8 \%$ by day 15 of hospitalization (Fig. 2). This decrease is represented by $y=$ $14.0+4.9 \exp (-0.2 \mathrm{x})$ by using least squares method ( $\mathrm{y}$ is defined as the cumulative incidence rate of true-blood cultures, $\mathrm{x}$ is defined as the day after admission), and according to this model, the cumulative incidence rate of blood cultures is prone to be stationary. Cumulative incidence of true-positive blood cultures obtained on the day of admis- sion and after admission is represented by $\mathrm{y}=10.7 \mathrm{x}+$ 499.4 by using linear regression ( $\mathrm{y}$ is defined as cumulative incidence of positive blood cultures, $x$ is defined as the day after admission; Fig. 3A). Cumulative incidence of blood cultures collected on the day of admission and after admission is represented by $y=111.5 x+2776.1$ using linear regression ( $\mathrm{y}$ is defined as cumulative incidence of blood cultures, $\mathrm{x}$ is defined as the day after admission; Fig. 3B). The true-positive and contamination rates of blood cultures obtained on the day of admission was $19.8 \%$ and $5.7 \%$, respectively (Fig. 4). The number of blood cultures for community-acquired group and hospital-acquired group was 2,774 sets collected from 2,206 patients and 5,666 sets collected from 1,911 patients, respectively. The median true-positive rate of blood cultures in the communityacquired group was not significantly higher than that in hospital-acquired group $(10.9 \%$ vs. $9.6 \%, p=0.77)$. 


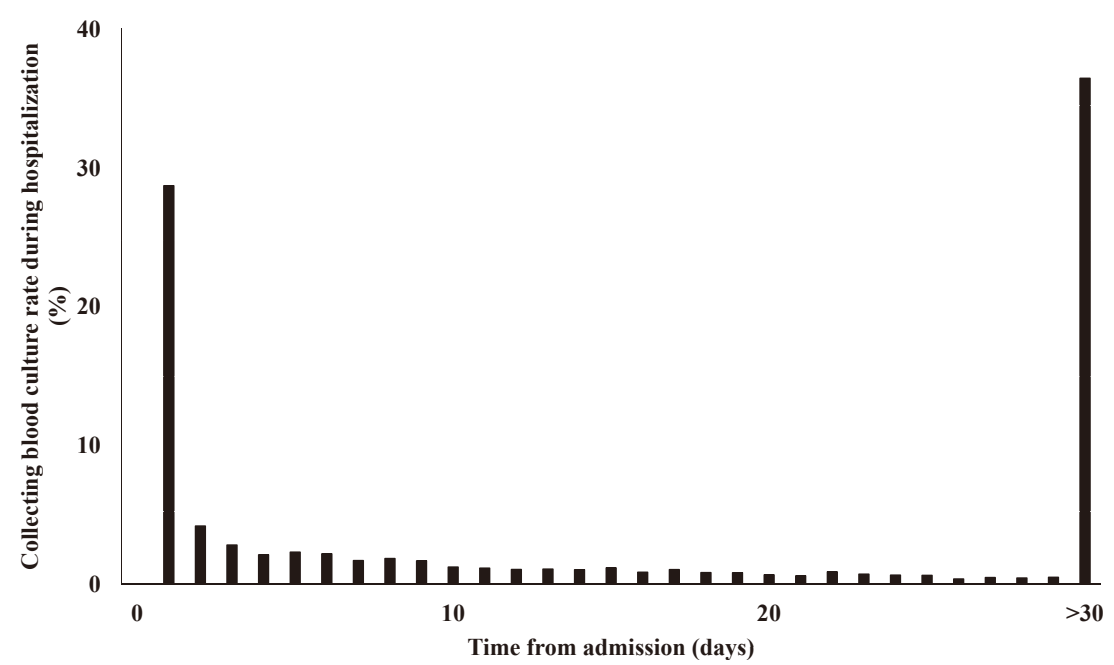

Fig. 1. Rate of collecting blood cultures during hospitalization.

The rate of total number of collections of blood culture during hospitalization shows a skewed distribution.

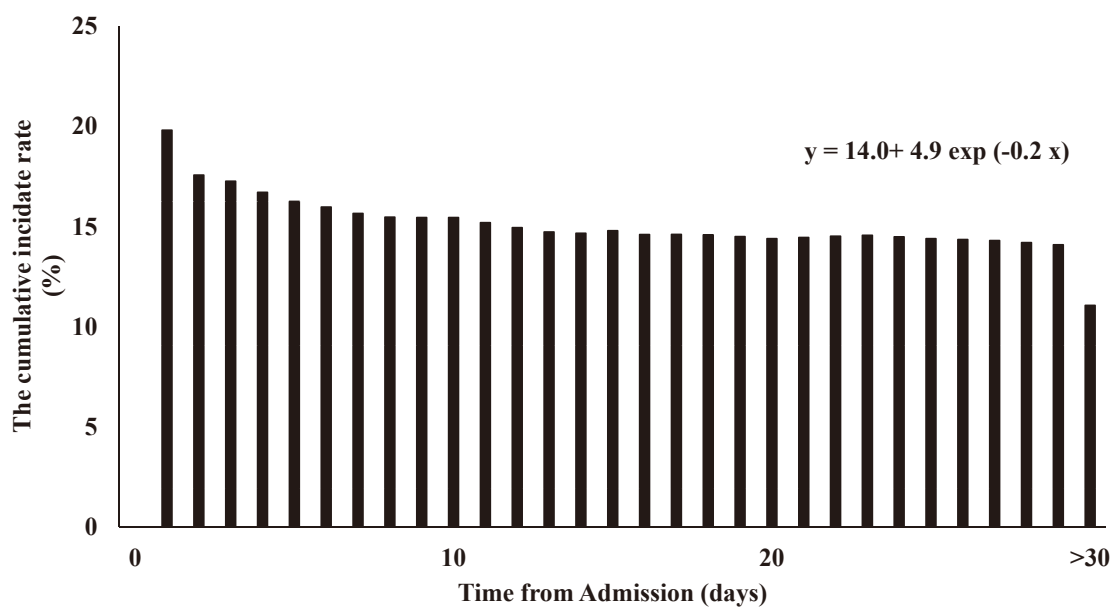

Fig. 2. Cumulative incidence rate of true-positive blood cultures during hospitalization.

The cumulative incidence rate of true-positive blood cultures is highest at the day of admission (25.5\%), and gradually decreases.

However, the median contamination rate of blood cultures in the community-acquired group was significantly higher than that in the hospital-acquired group $(4.1 \%$ vs. $1.9 \%, p=$ $0.029)$.

\section{Discussion}

We found that (1) the number of blood cultures per 1,000 patient-days at our hospital was lower than those recommended by Cumitech 1C, Blood Cultures (Baron et al. 2005), (2) the cumulative incidence rate of true-positive blood cultures gradually decreased during hospitalization and (3) the median contamination rate of blood cultures in the community-acquired group was significantly higher than that in the hospital-acquired group.

The number of blood cultures per 1,000 patient-days at our hospital (range 28.6-56) was lower than the number recommended by the Cumitech guidelines (range 103-188).
In previous reports from Japan, results were 10.4-64.2 per 1,000 patient-days (Ohmagari et al. 2012), 12.3-15.4 per 1,000 patient-days (Nagao 2013), and 9.3-22.4 per 1,000 patient-days (Shigoka et al. 2016).

This difference can be explained by differences in the average length of stay, patient demographics, and the healthcare system, especially medical insurance, between Japan and the USA (Ohmagari et al. 2012). Patient-days is defined as the average length of stay $\times\{$ (admissions + discharges) $/ 2\}$ in Japan. In a previous report, average length of stay was calculated as; average length of stay $=$ total number of bed days (inpatient) in the year/number of discharges and deaths in the same year (Somotun et al. 2017). Since the median number of admissions and discharges per year is quite similar in general and in our hospital, we can approximate patient-days as the average length of stay $x$ total number of admissions. Therefore, based on this 


\section{A}
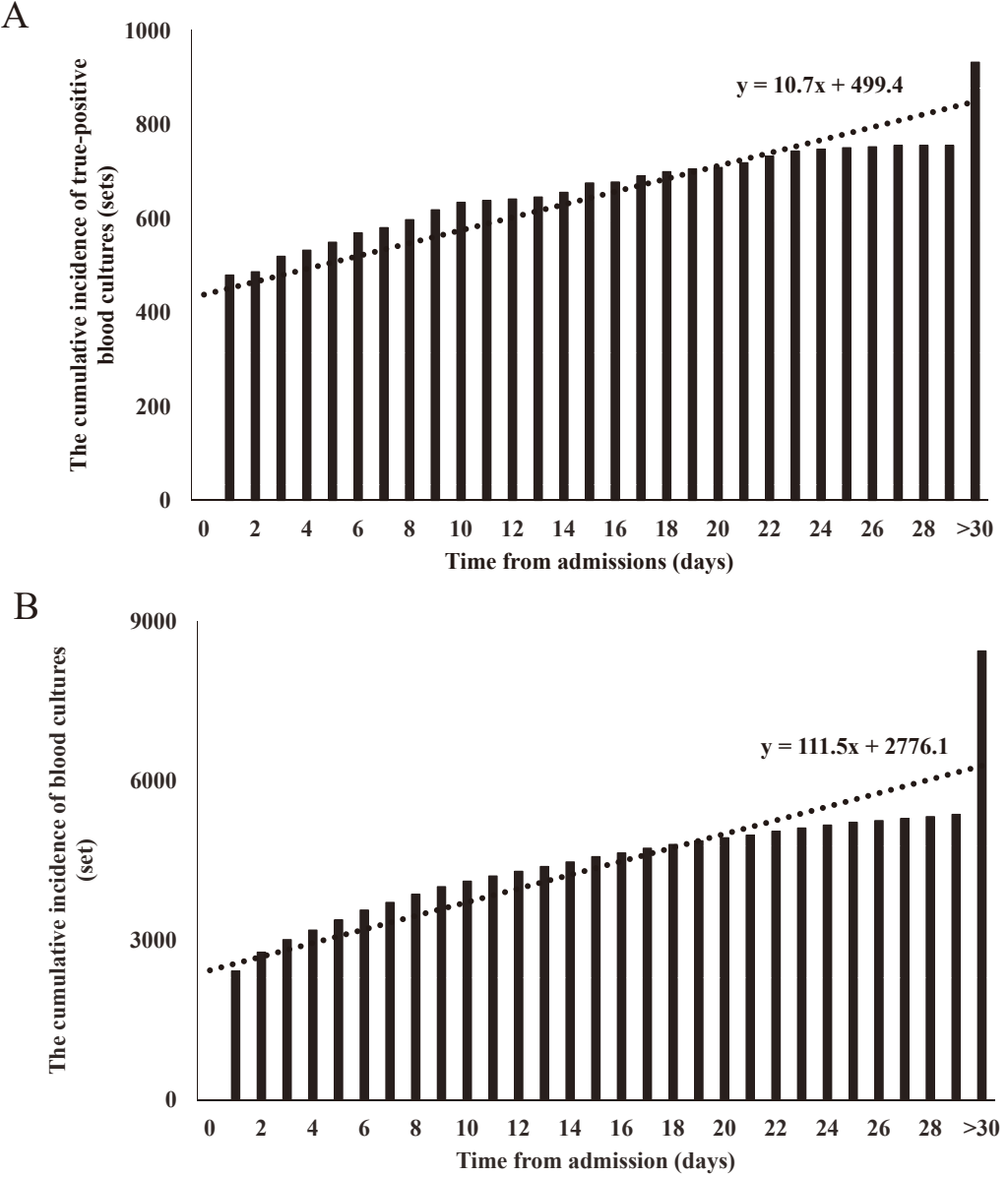

Fig. 3. Cumulative incidence rate of blood cultures.

A. Cumulative incidence of true-positive blood cultures. B. Cumulative incidence of blood cultures. The slope of number of blood cultures is greater than that of number of positive blood cultures.

approximation, the number of blood cultures per 1,000 admissions is defined as the number of blood cultures per 1,000 patient-days $\times$ the average length of stay, as follows:

Number of blood cultures per 1,000 patient-days $=$ (total number of blood cultures/cumulative number of patients admitted) $\times 1,000$

$\rightarrow$ Number of blood cultures per 1,000 patient-days $=$ total number of blood cultures/ \{the average length of stay $x$ $($ admissions + discharges/2) $\} \times 1,000$

$\rightarrow$ Number of blood cultures per 1,000 patient-days $=$ \{total number of blood cultures/(the average length of stay $\times$ admissions $)\} \times 1,000[1]$

$\rightarrow$ Number of blood cultures per 1,000 patient-days $\times$ the average length of stay $=$ Number of blood cultures per 1,000 admissions [2]

Length of stay is affected by age, morbidity, hospital size, and the type of hospital. Thus, length of stay varies substantially among different countries (Kuster et al. 2008). The average length of stay in our hospital in 2010 was 15.3 days, whereas the average length of stay in the USA in 2012 was 4.5 days (Weiss et al. 2014). Therefore, using the formula above [2] and number of blood cultures per 1,000 patient-days recommended by the Cumitech guidelines (range 103-188), the number of blood cultures per 1,000 admissions in the USA would be 463-846. This number is similar to that in our hospital (range 404.4-894.6). Moreover, the number of blood cultures obtained on a given day is largest on the day of admission, gradually decreasing on subsequent days of hospitalization (Fig. 1). Within a period not exceeding the first 30 days from admission, the number of blood cultures is dependent on the early phase of admission, and thus the total number of blood cultures is less affected by the factor of length of stay. On the other hand, the factor of patient-days is affected by and dependent on length of stay (Novis et al. 2001; Filius et al. 2005). The number of blood cultures per 1,000 patient-days can therefore be assumed to decrease as the average length of stay increases. Thus, we believe that using the number of blood cultures per 1,000 admissions as an indicator is more appropriate for international comparison, taking into consideration the difference in the average length of stay.

The median true-positive rate of blood cultures at our hospital was similar to previous studies (Baron et al. 2005; Nannan Panday et al. 2019). However, these rates vary in the hospital settings. The cumulative incidence rate of truepositive blood cultures gradually decreased during hospital- 
A

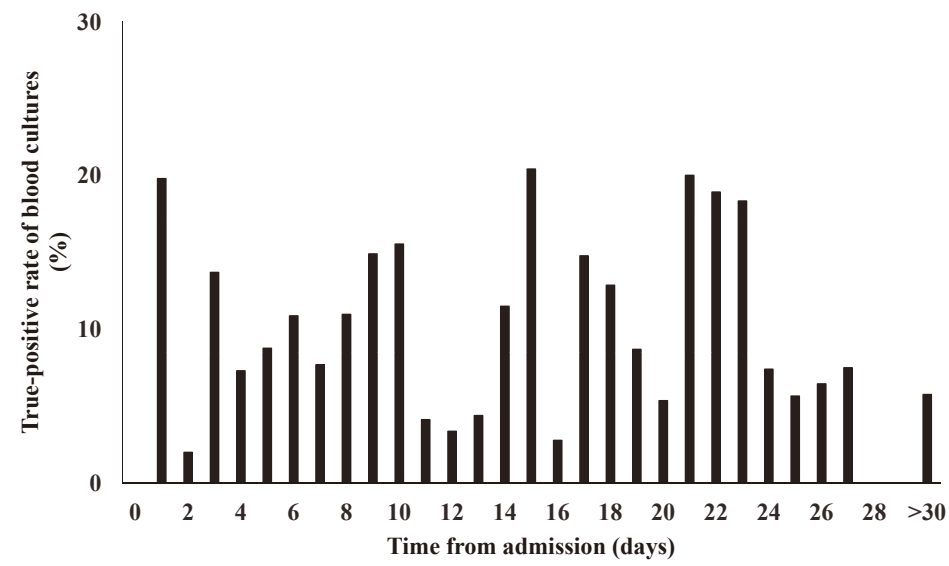

B

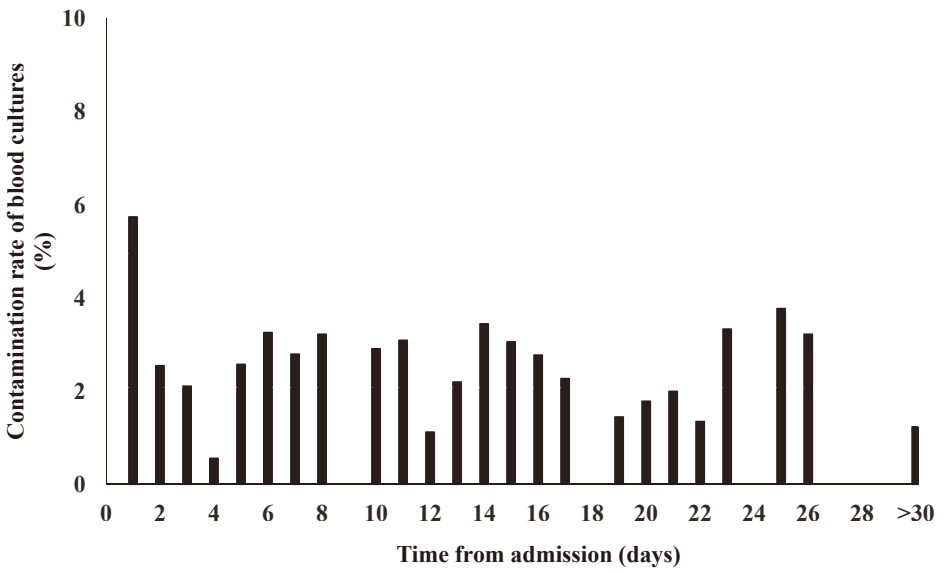

Fig. 4. Positive rate of blood cultures during hospitalization.

A. The true-positive rate of blood cultures. B. The contamination rate of blood cultures.

ization (Fig. 2), because the slope of cumulative incidence of blood cultures collected on the day of admission and after admission is greater than that of cumulative true-positive blood cultures on the day of admission and after admission (Fig. 3). This means that it is possible that the difference in the total length of stay (measured in days of admission) was related to the difference of the true-positive rate of blood cultures in the hospital setting. Therefore, although the true-positive rate of blood cultures is considered as a performance indicator for the practice of blood culture, this parameter may be influenced by length of stay.

It is reported that $<3 \%$ blood culture contamination is the benchmark for an acceptable contamination rate (Doern et al. 2019). Halstead et al. (2020) have proposed a benchmark of $1.5-2.0 \%$ is achievable. The median contamination rate of blood cultures at our hospital (range 2.2-2.9\%) was similar to previous studies. However, the median contamination rate of blood cultures in the community-acquired group was significantly higher than that in the hospitalacquired group $(4.1 \%$ vs. $1.9 \%)$. This difference may be due to inadequate blood culture techniques as medical staff performed many medical interventions to the patients on the day of admission. We could not analyze the difference of the groups due to insufficient data on patient population and medical departments. A further analysis using suffi- cient data would be necessary to clarify the association between the contamination rate and the hospital settings.

Contaminated blood cultures result in delayed diagnosis, increased length of stay, unnecessary use of antimicrobial agents and additional health care cost. Therefore, blood culture, in particular if it is obtained within 48 hours after admission, should be obtained using continuous monitoring, appropriate feedback and continued education along with using sterile gloves, collection kits and diversion devices (Halstead et al. 2020).

Our study has several limitations. First, this study was conducted in a single institution and is retrospective in design; thus, the sample size was small. Regardless, the number of blood cultures at our hospital being similar to that at other hospitals in Japan leads us to believe that our data could be valid for Japan in general to some extent. Second, since we did not collect any clinical data except for the days of admission and timing of collecting blood cultures, we were not able to compare other differences in medical situations between Japan and the USA. Third, an adequate volume of blood per bottle is the important parameter for the detection of bloodstream infections. The Clinical and Laboratory Standards Institute (CLSI) guidelines recommends to take four bottles with a blood volume of 8 to $10 \mathrm{~mL}$ each (two sets) (CLSI 2007). Although we 
were unable to assess this information, we considered that volume of blood per bottle is valid to some extent, because the positive rate of blood cultures and contamination rates in our study are comparable with previous studies.

In conclusion, the number of blood cultures per 1,000 admissions in the USA was from 463 to 846 , similar to that observed in our hospital. Therefore, there is a possibility that the number of blood cultures per 1,000 admissions is a more appropriate factor to evaluate than per patient-days, taking into consideration the difference in average length of stay between countries. Moreover, the true-positive and contamination rates of blood cultures in our hospital are valid.

\section{Conflict of Interest}

The authors declare no conflict of interest.

\section{References}

Baron, E.J., Weinstein, M.P., Dunne, W.M., Yagupsky, P., Welch, D.F. \& Wilson, D.M. (2005) Cumitech 1C: Blood Cultures $I V$, American Society for Microbiology. Washington, DC.

Bate, D.W., Cook, E.F, Goldman, L. \& Lee, T.H. (1990) Predicting bacteremia in hospitalized patients. A prospectively validated model. Ann. Intern. Med., 113, 495-500.

Chen, A.I., Bilker, W.B., Hamilton, K.W., O’Donnell, J.A. \& Nachamkin, I. (2018) Blood culture utilization at an academic hospital: addressing a gap in benchmarking. Infect. Control Hosp. Epidemiol., 39, 1353-1359.

Clinical and Laboratory Standards Institute (CLSI) (2007) Principles and procedures for blood cultures; approved guideline, CLSI document M47-A, Clinical and Laboratory Standards Institute, Wayne, P.A.

Doern, G.V., Carroll, K.C., Diekema, D.J., Garey, K.W., Rupp, M.E., Weinstein, M.P. \& Sexton, D.J. (2019) Practical guidance for clinical microbiology laboratories: a comprehensive update on the problem of blood culture contamination and a discussion of methods for addressing the problem. Clin. Microbiol. Rev., 33, e0009-19.

Filius, P.M., Liem, T.B., van der Linden, P.D., Janknegt, R., Natsch, S., Vulto, A.G. \& Verbrugh, H.A. (2005) An additional measure for quantifying antibiotic use in hospitals. $J$. Antimicrob. Chemother, 55, 805-808.

Halstead, D.C., Sautter, R.L., Snyder, J.W., Crist, A.E. \& Nachamkin, I. (2020) Reducing blood culture contamination rates: experiences of four hospital systems. Infect. Dis. Ther., 9, 389-401.

Kanda, Y. (2013) Investigation of the freely available easy-to-use software 'EZR'for medical statistics. Bone Marrow Transplant., 48, 452-458.

Kim, E.C., Shin, J.H., Kim, S., Lee, N.Y., Cho, J., Koo, S.H., Ryoo, N.H. \& Joo, S.I. (2012) Number of blood cultures per 1,000 patient days at university-affiliated hospitals in Korea. Korean J. Clin. Microbiol., 15, 67-69.

Kuster, S.P., Ruef, C., Ledergerber, B., Hintermann, A., Deplazes, C., Neuber, L. \& Weber, R. (2008) Quantitative antibiotic use in hospitals: comparison of measurements, literature review, and recommendations for a standard of reporting. Infection, 36, 549-559.

Nagao, M. (2013) A multicentre analysis of epidemiology of the nosocomial bloodstream infections in Japanese university hospitals. Clin. Microbiol. Infect., 19, 852-858.

Nannan Panday, R.S., Wang, S., van de Ven, P.M., Hekker, T.A.M., Alam, N. \& Nanayakkara, P.W.B. (2019) Evaluation of blood culture epidemiology and efficiency in a large European teaching hospital. PLoS One, 14, e0214052.

Novis, D.A., Dale, J.C., Schifman, R.B., Ruby, S.G. \& Walsh, M.K. (2001) Solitary blood cultures: a College of American Pathologists Q-probes study of 132,778 blood culture sets in 333 small hospitals. Arch. Pathol. Lab. Med., 125, 12901294.

Ohmagari, N., Takakura, S., Matsumura, Y., Sugiyama, T., Takeshita, N., Takahashi, M., Ainoda, Y., Goto, A., Chibana, N., Ohshiro, T., Uno, K., Nakayama, A., Kubo, K. \& Ikeda, N. (2012) A questionnaire survey of blood culture among Japanese hospitals: a pilot study. The Journal of Japanese Society for Clinical Microbiology, 22, 13-19 (in Japanese).

Shigoka, T., Buya, Y., Igawa, S., Kobayashi, H., Miyamae, M. \& Yasuno, A. (2016) Cultivation of multiple blood culture sets and consequent improvements via promotional activities by the infection control team in our hospital. Japanese Journal of Infection Prevention and Control, 31, 382-389 (in Japanese).

Somotun, O.A.A., Osungbade, K.O., Akinyemi, O.O., Obembe, T.A. \& Adeniji, F.I. (2017) What factors influence the average length of stay among stroke patients in a Nigerian tertiary hospital? Pan Afr. Med. J., 26, 228.

Weiss, A.J. \& Elixhauser, A. (2014) Overview of hospital stays in the United States, 2012: statistical brief \#180. In Healthcare Cost and Utilization Project (HCUP), Statistical Brief [Internet], Agency for Healthcare Research and Quality, Rockville, M.D. 